\title{
SPECTRAL OPERATORS ON LOCALLY CONVEX SPACES
}

\author{
BY C. IONESCU TULCEA \\ Communicated by Walter Rudin, September 12, 1960
}

1. Let $C$ be the complex plane, $S(C)$ the tribe of all Borel parts of $C, B^{\infty}(C)$ the algebra of bounded complex-valued Borel measurable functions defined on $C$ and $M^{1}(C)$ the set of bounded complex Radon measures on $C$. Let $E$ be a locally convex space ${ }^{1}$ which is separated, quasi-complete and barrelled. A family $\mathfrak{F}=\left(m_{x, x^{\prime}}\right)_{x \in E, x^{\prime} \in E^{\prime}}$ of measures belonging to $M^{1}(C)$ is called a spectral family on $C$ if there exists a representation $f \rightarrow U_{\mathscr{F}, f}$ of the algebra $B^{\infty}(C)$ into the algebra ${ }^{1}$ $L(E, E)$ mapping 1 onto $I$ and satisfying the equations $\int_{C} f d m_{x, x^{\prime}}$ $=\left\langle U_{\mathscr{F}, f} x, x^{\prime}\right\rangle$ for all $f \in B^{\infty}(C), x \in E, x^{\prime} \in E^{\prime}$. By $P_{\mathscr{F}}$ we denote the spectral measure defined on $S(C)$ by the equations $P_{\mathscr{F}}(\sigma)=U_{\mathfrak{F}, \phi_{\sigma}}$ ( $\phi_{\sigma}$ is the characteristic function of $\sigma$ ). A linear mapping $T$ of (the vector space) $D_{T} \subset E$ into $E$ commutes with $\mathcal{F}$ if $T U_{\mathscr{F}, f} \supset U_{\mathscr{F}, f} T$ for all $f \in B^{\infty}(C)$.

Let $T$ be a linear mapping of $D_{T} \subset E$ into $E$. We say that $\lambda \in \hat{C}$ (= the one point compactification of $C$ ) belongs to the resolvent set $r(T)$ of $T$ if there is a neighborhood $V$ of $\lambda$ such that: (i) $z I-T$ is a one-to-one mapping of $D_{T}$ onto $E$ and $(z I-T)^{-1} \in L(E, E)$ for each $z \in V-\{\infty\}$; (ii) $\left\{(z I-T)^{-1} \mid z \in V-\{\infty\}\right\}$ is a bounded part of $L(E, E)$. The set $\operatorname{sp}(T)=\hat{C}-r(T)$ is the spectrum of $T$. If $\operatorname{sp}(T) \boxplus \infty$ we say that $T$ is regular.

By an admissible set we mean a directed (for $C$ ) set of closed parts of $C$ whose union is $C$, having a countable cofinal part and containing with $A \subset C$ every closed part of $A$. We denote below by $\mathfrak{e}_{0}$ and $\mathfrak{e}_{1}$ the admissible set of all compact parts of $C$ and all closed parts of $C$, respectively. Let $\mathcal{C}$ be an admissible set and $T$ a closed linear mapping of $D_{T} \subset E$ into $E$. We say that $T$ is a e-spectral operator if there is a spectral family $\mathcal{F}$ on $C$ such that:

(DI) $T$ commutes with $F$;

(DII) $T U_{\mathcal{F}, f} \in L(E, E)$ for each $f \in B^{\infty}(C)$ whose support is compact and belongs to $\mathfrak{C}$;

$\left(D_{\text {III }}\right) \operatorname{sp}\left(T_{\sigma}\right) \subset \sigma^{-}$for every $\sigma \in \mathbb{C}$.

${ }^{1} E$ barrelled means that every weakly bounded part of the dual space $E^{\prime}$ is equicontinuous; $E$ quasi-complete means that every bounded closed part of $E$ is complete. $L(E, E)$ is the algebra of all linear continuous mappings of $E$ into $E$ endowed with the topology of uniform convergence on the bounded parts of $E$.

2 For a set $A \subset C$ we denote by $A^{-}$the closure of $A$ in $\hat{C}$. 
(For $\sigma \in S(C)$ we denote by $T_{0}$ the mapping $x \rightarrow T x$ of $D_{T} \cap E_{\sigma}$ into $E_{\sigma}$, where $E_{\sigma}=P_{\mathcal{F}}(\sigma)(E)$.)

THEOREM 1. Let $\mathfrak{e}$ be an admissible set and $T$ a closed linear mapping of $D_{T} \subset E$ into $E$. Then there is at most one spectral family on $C$ satisfying $\left(\mathrm{D}_{\mathrm{I}}\right),\left(\mathrm{D}_{\mathrm{II}}\right)$ and $\left(\mathrm{D}_{\mathrm{III}}\right)$.

For a e-spectral operator $T$ we shall denote by $F_{T}$ the unique spectral family on $C$ satisfying $\left(D_{\mathrm{I}}\right),\left(D_{I I}\right)$ and $\left(D_{I I I}\right)$.

Theorem 2. Let $T$ be a e-spectral operator. Then every $A \in L(E, E)$ commuting with $T$ commutes with $\mathfrak{F}_{T}$.

Let now $\mathcal{C}$ be an admissible set of parts of $C$ and $\mathcal{F}=\left(m_{x, x^{\prime}}\right)_{x \in E, x^{\prime} \in E^{\prime}}$ a spectral family on $C$. Consider the following property concerning $\mathcal{F}: P \mathfrak{C})$. Given $x \in E, x^{\prime} \in E^{\prime}$ there is $\sigma\left(x, x^{\prime}\right) \in \mathfrak{C}$ such that the supports of the measures $m_{Q x, x^{\prime}}$ are contained in $\sigma\left(x, x^{\prime}\right)$ for all $Q \in L(E, E)$ commuting with $\mathcal{F}$.

THEOREM 3. Let $T$ be a e-spectral operator and suppose that $\mathfrak{F}_{T}$ has property $P \mathcal{C})$. Then $\operatorname{sp}\left(T_{\sigma}\right) \subset \sigma^{-}$for all $\sigma \in \mathcal{C}_{1}$.

THEOREM 4. Let $T$ be a e-spectral operator. Then ${ }^{3}:(4.1) S\left(F_{T}\right)$ $C \mathrm{sp}(T) .(4.2)$ If $\mathfrak{F}_{T}$ has property $\left.P \mathfrak{C}\right)$ then $S\left(\mathcal{F}_{T}\right)^{-}=\operatorname{sp}(T)$.

2. We say that an operator $S \in L(E, E)$ is scalar if there is a spectral family $\mathcal{F}=\left(m_{x, x^{\prime}}\right)_{x \in E, x^{\prime} \in E^{\prime}}$ on $C$ of measures with compact support such that $\int_{C} z d m_{x, x^{\prime}}=\left\langle S x, x^{\prime}\right\rangle$ for all $x \in E, x^{\prime} \in E^{\prime}$; we write in this case $S=U_{\mathfrak{F}, z}$. An operator $Q \in L(E, E)$ is quasi-nilpotent if $\lim _{n \rightarrow \infty}\left|\left\langle Q^{n} x, x^{\prime}\right\rangle\right|^{1 / n}=0$ for all $x \in E, x^{\prime} \in E^{\prime}$.

THEOREM 5. (5.1) Let $T \in L(E, E)$ be a $\mathfrak{C}_{0}$-spectral operator and suppose that $\mathfrak{F}_{T}$ has property $\left.P \mathcal{C}_{0}\right)$. Then $T=U_{\mathfrak{F}_{T, z}}+Q$, where $Q$ is quasinilpotent, and $T, U F_{T, z}, Q$ commute. Further if $T=S+R$ where $S$ is scalar, $R$ quasi-nilpotent and where $T, S, R$ commute, then $S=U F_{T, z}$ and $R=Q$. (5.2) Let $\mathcal{F}$ be a spectral family on $C$ of measures with compact support and $Q$ a quasi-nilpotent operator commuting with $F$. Then $T=U_{\mathcal{F}, z}+Q$ is a $\mathfrak{C}_{0}$-spectral operator and $\mathfrak{F}=\mathcal{F}_{T}$.

3. In what follows we denote by $\Phi$ an arbitrary directed family of closed barrelled subspaces of $E$ having the properties: (i) the set $E_{0}$ $=\bigcup_{F \in \Phi} F$ is dense in $E$; (ii) a linear mapping $T$ of $E_{0}$ into $E_{0}$ verifying the relations $T(F) \subset F$ for all $F \in \Phi$ is continuous if $T_{F}\left(T_{F}\right.$ is the mapping $x \rightarrow T x$ of $F$ into $F$ ) is continuous for all $F \in \Phi$; (iii) given

\footnotetext{
${ }^{8}$ We denote by $S\left(\mathcal{F}_{T}\right)$ the closure in $C$ of the union of the supports of the measures
} belonging to $\mathcal{F}_{T}$. 
$x \in E$ and $x^{\prime} \in E^{\prime}$ there is $x_{0} \in E_{0}$ verifying the equations $\left\langle T x, x^{\prime}\right\rangle$ $=\left\langle T x_{0}, x^{\prime}\right\rangle$ for each $T \in L(E, E)$ such that $T(F) \subset F$ for all $F \in \Phi$. Given $\Phi$ let $L_{\Phi}(E, E)$ be the set of all $T \in L(E, E)$ such that: (j) $T(F)$ $\subset F$ for all $F \in \Phi$; (jj) $T_{F}$ is regular for all $F \in \Phi$ and $\operatorname{sp}\left(T_{F^{\prime}}\right) \subset \operatorname{sp}\left(T_{F^{\prime \prime}}\right)$ $\subset \operatorname{sp}(T)$ if $F^{\prime}, F^{\prime \prime} \in \Phi, F^{\prime} \subset F^{\prime \prime}$. For $T \in L_{\Phi}(E, E)$ we write $A(T)$ $=\bigcup_{F \in \Phi} \operatorname{sp}\left(T_{F}\right)$.

Theorem 6. If $T \in L_{\Phi}(E, E)$ then $\operatorname{sp}(T)=A(T)$ -

THEOREM 7. If $T \in L_{\Phi}(E, E)$ then there exists a unique continuous representation $\tilde{f} \rightarrow \tilde{f}(T)$ of $H(A(T))$ into $L(E, E)$ having the properties: (7.1) $\tilde{1}(T)=I ;(7.2) \quad \tilde{z}(T)=T$. Further $\tilde{f}(T) \in L_{\Phi}(E, E)$ and $\operatorname{sp}(\tilde{f}(T))=f(A(T))-(f$ is an element in the equivalence class $\tilde{f})$.

Let $T \in L(E, E)$ be a $\mathfrak{C}_{0}$-spectral operator. Suppose that $\mathfrak{F}_{T}$ $=\left(m_{x, x^{\prime}}\right)_{x \in E, x^{\prime} \in E^{\prime}}$ has property $\left.P \mathcal{C}_{0}\right)$ and let $\left.\Phi=\left(E_{\sigma}\right)_{\sigma \in \mathfrak{C}_{0}}\right)$. Then $\Phi$ has the properties (i), (ii), (iii) and $T \in L_{\Phi}(E, E)$. Moreover:

THEOREM 8. The operator $\tilde{f}(T)$ is $\mathfrak{C}_{1}$-spectral for each $\tilde{f} \in H(A(T))$ and

$$
\left\langle f(T) x, x^{\prime}\right\rangle=\sum_{j=0}^{\infty} \frac{1}{j !} \int_{C} f^{(j)} d m_{Q^{\prime}, x^{\prime}}, \quad \text { for } x \in E, x^{\prime} \in E^{\prime},
$$

where $Q$ is the quasi-nilpotent part of $T$. The series (1) converges $a b$ solutely and uniformly for given $x \in E$ and $x^{\prime} \in A$ ( $A$ is an arbitrary equicontinuous part of $E^{\prime}$ ).

4. Let $\mathfrak{C}$ be an admissible set, $(\sigma(n))$ an increasing sequence of compact parts belonging to $\mathcal{C}$ whose union is $C, T: D_{T} \rightarrow E$ a $\mathcal{C}$ spectral operator, $\mathfrak{F}_{T}=\left(m_{x, x^{\prime}}\right)_{x \in E, x^{\prime} \in E^{\prime}}$ and $E_{\infty}=U E_{\sigma(n)}$. Let $T_{\infty}$ be the restriction of $T$ to $E_{\infty} \subset D_{T}$ and $\mathcal{F}_{T}^{\infty}=\left(m_{x, x^{\prime}}^{\infty}\right)_{x \in E_{\infty}, x^{\prime} \in E_{\infty}^{\prime}}$. Here $E_{\infty}$ is endowed with the topology, inductive limit of the topologies of the subspaces $E_{\sigma(n)}$ of $E$, and, for $x \in E_{\sigma(n)} \subset E_{\infty}$ and $x^{\prime} \in E_{\infty}^{\prime}, m_{x, x^{\prime}}^{\infty}=m_{x, y^{\prime}}$ if $y^{\prime} \in E^{\prime}$ is such that $x_{E_{\sigma(n)}}^{\prime}=y_{E_{\sigma(n)}}^{\prime}$.

TheOREM 9. (9.1) $T_{\infty}$ is a $\mathcal{C}_{1}$-spectral operator, $\mathfrak{F}_{T_{\infty}}=\mathfrak{F}_{T}^{\infty}$ and $\mathfrak{F}_{T_{\infty}}$ has property $P \Sigma)(\Sigma$ is the smallest admissible set containing $(\sigma(n)))$. (9.2) $T$ is the closure of $T_{\infty}$. (9.3) $\operatorname{sp}\left(T_{\infty}\right)=S\left(\mathcal{F}_{T}\right)^{-}$.

Further $A \in L(E, E)$ commutes with $T$ if and only if $A\left(E_{\infty}\right) \subset E_{\infty}$ and $A_{E_{\infty}}$ commutes with $T_{\infty}$. Also $T$ is "scalar" if and only if $T_{\infty}$ is scalar; if $T$ is "scalar" and $f$ is such that $\phi_{\sigma(n)} f \in B^{\infty}(C)$ for all $n$ then $f(T)_{\infty}=f\left(T_{\infty}\right)$.

"For the definition of $H(A), A \subset C$ (endowed with the "van Hove topology"), see for instance [5] (where $A$ is supposed compact) and [4, pp. 255-256]. 
5. The subject matter of this note has been suggested by [2] and by $[1 ; 3]$. The Theorems $1,2,5$ and 8 are essentially generalizations of the corresponding results in $[2 ; 3]$. The results of paragraph 4 show some of the relations between the unbounded spectral operators defined in [1] and the (everywhere defined continuous) spectral operators defined above. The definition of the spectrum and of the quasinilpotent operator were suggested by definitions given in $[5 ; 6]$, respectively.

\section{REFERENCES}

1. W. G. Bade, Unbounded spectral operators, Pacific J. Math. vol. 4 (1954) pp. $373-392$.

2. N. Dunford, Spectral operators, Pacific J. Math. vol. 4 (1954) pp. 321-354.

3. N. Dunford and J. T. Schwartz, Spectral operators, Unpublished manuscript.

4. A. Grothendieck, Espaces vectoriels topologiques, São Paulo, 1958.

5. L. Waelbroeck, Le calcul symbolique dans les algèbres commutatives, J. Math. Pures Appl. vol. 43 (1954) pp. 147-186.

6. J. H. Williamson, Linear transformations in arbitrary linear spaces, J. London Math. Soc. vol. 28 (1953) pp. 203-210.

YALE UNIVERSITY 